\title{
Limitation in tidal volume expansion partially determines the intensity of physical activity in COPD
}

\author{
Eleni A. Kortianou, ${ }^{1,2,3}$ Andrea Aliverti, ${ }^{4}$ Zafeiris Louvaris, ${ }^{1}$ Maroula Vasilopoulou, ${ }^{1}$ Ioannis Nasis, ${ }^{1}$ \\ Andreas Asimakos, ${ }^{2}$ Spyros Zakynthinos, ${ }^{2}$ and Ioannis Vogiatzis ${ }^{1,2}$ \\ ${ }^{1}$ National and Kapodistrian University of Athens, Department of Physical Education and Sports Sciences, Athens, Greece; \\ ${ }^{2}$ National and Kapodistrian University of Athens, 1st Department of Critical Care Medicine, Evangelismos Hospital, Athens, \\ Greece; ${ }^{3}$ Technological Educational Institute of Central Greece, Department of Physiotherapy, Lamia, Greece; ${ }^{4}$ Dipartimento \\ di Elettronica, Informazione e Bioingeneria, Politecnico di Milano, Italy
}

Submitted 3 April 2014; accepted in final form 12 November 2014

In patients with chronic obstructive pulmonary disease (COPD), reduced levels of daily physical activity are associated with the degree of impairment in lung, peripheral muscle, and central hemodynamic function. There is, however, limited evidence as to whether limitations in tidal volume expansion also, importantly, determine daily physical activity levels in COPD. Eighteen consecutive patients with COPD [ 9 active (forced expiratory volume in $1 \mathrm{~s}, \mathrm{FEV}_{1}: 1.59 \pm$ 0.64 1) with an average daily movement intensity $>1.88 \mathrm{~m} / \mathrm{s}^{2}$ and 9 less active patients $\left(\mathrm{FEV}_{1}: 1.16 \pm 0.41 \mathrm{l}\right)$ with an average intensity $<$ $1.88 \mathrm{~m} / \mathrm{s}^{2}$ ] underwent a 4-min treadmill test at a constant speed corresponding to each indi-vidual patient's average movement intensity, captured by a triaxial accelerometer during a preceding 7day period. When chest wall volumes, captured by optoelectronic plethysmography, were ex-pressed relative to comparable levels of minute ventilation (ranging between $14.5 \pm 4.3$ to $33.5 \pm 4.41 / \mathrm{min}$ ), active patients differed from the less active ones in terms of the lower increase in end-expiratory chest wall volume (by $0.15 \pm 0.17$ vs. $0.45 \pm$ 0.211 ), the greater expansion in tidal volume (by $1.76 \pm 0.58$ vs. $1.36 \pm$ 0.241 ), and the larger inspiratory reserve chest wall volume (IRVcw: by $0.81 \pm 0.25$ vs. $0.39 \pm 0.271)$. IRVcw $\left(r^{2}=0.420\right)$, expiratory flow $\left(r^{2}\right.$ change $\left.=0.174\right)$, and Borg dyspnea score $\left(r^{2}\right.$ change $=$ 0.123 ) emerged as the best contributors, accounting for $71.7 \%$ of the explained variance in daily movement intensity. Patients with COPD exhibiting greater ability to expand tidal volume and to maintain adequate inspiratory reserve volume tend to be more physically active. Thus interventions aiming at mitigating restrictions on operational chest wall volumes are expected to enhance daily physical activity levels in COPD.

IN PATIENTS WITH CHRONIC OBSTRUCTIVE PULMONARY DISEASE (COPD), exercise limitation is multifactorial, involving ventilatory, gas exchange, cardiovascular, and lower-limb muscle dysfunctional factors (32). Similarly, several reports in the past few years demonstrated that, in patients with COPD, reduced levels of daily physical activity are related to important physiological limitations imposed by impaired lung and peripheral muscle function $(6,9,15,26,33)$.

Address for reprint requests and other correspondence: I. Vogiatzis, Thorax Foundation, 3str Ploutarhou 10675, Athens, Greece (e-mail: gianvog@phed. uoa.gr).
Focusing on the impact of lung dysfunction on daily physical activity levels in COPD, the study by Garcia-Rio and colleagues (9) reported that the intensity of daily physical activity is associated with the peak magnitude of dynamic lung hyperinflation that was, albeit, recorded at the limit of tolerance of a maximal exercise test and not at the intensity of movement commonly adopted by each individual in his/her real daily life. Furthermore, a more detailed inspection of this study (9) indicated that a large fraction of patients $(>25 \%)$ who were minimally hyperinflated at maximal exercise [i.e., exhibiting an increase in end-expiratory lung volume $($ EELV) $<150 \mathrm{ml}]$ demonstrated a significant variability in the intensity of daily physical activity ranging between 100 and 400 vector magnitude units (VMUs). Similarly, patients exhibiting profound dynamic lung hyperinflation (i.e., an increase in EELV >300 $\mathrm{ml}$ ) also exhibited considerable variation in daily physical activity levels that ranged between 100 and 300 VMUs. These observations raise the question of the extent to which dynamic lung hyperinflation and the concurrent limitations in tidal volume expansion restrict the intensity of daily physical activity in patients with different degrees of manifestation of dynamic lung hyperinflation.

Indeed, well-controlled laboratory studies using cycle ergometry as the mode of exercise have shown that, irrespective of the magnitude and pattern of dynamic hyperinflation (2, 29, 31 ), the magnitude of limitations in both tidal and inspiratory reserve volumes $(7,8,19-22)$ importantly determine exercise capacity in COPD. Interestingly, these data have been identified during maximal cycle ergometry testing rather than submaximal activities mimicking real-life activities such as walking. Hence, to get a global view of the respiratory factors limiting the intensity of daily physical activity in COPD, one should determine the magnitude and pattern of dynamic hyperinflation in conjunction with indexes of mechanical limitation of all breathing volumes during modalities and intensities of physical activities, which resemble those in daily life.

Therefore, the purpose of the present study was to investigate whether differences in physical activity levels commonly adopted by individual patients with COPD in daily life compared with well-controlled laboratory environments are partly attributed to differences in the magnitude of mechanical limitation to total and compartmental (rib cage and abdominal) chest wall volumes. To accomplish our goal, we employed optoelectronic plethysmography (OEP), allowing breath-bybreath measurements of end-expiratory, end-inspiratory, and inspiratory reserve chest wall volumes. 
Several studies in the past $(2,4,29,31)$ have utilized OEP to assess breath-by-breath changes in end-expiratory chest wall volume as an index of the magnitude of exercise-induced dynamic chest wall hyperinflation in patients with COPD. In the present study, chest wall volumes were recorded during walking on a treadmill at a speed corresponding to the average intensity of daily activity of each individual patient with COPD, which was captured by an accelerometer during a period of seven preceding consecutive days. On the basis of the median daily movement intensity $\left(1.8-1.9 \mathrm{~m} / \mathrm{s}^{2}\right)$ previously documented by our group and others $(11,15,23)$, patients were classified as active or less physically active. It was reasoned that less active patients would differ from the more active ones in terms of the degree of limitation in tidal volume expansion and the ability to maintain adequate inspiratory reserve during daily activities.

\section{MATERIALS AND METHODS}

Study design. The present study was part of a broader experimental project with a twofold purpose in mind, namely to investigate associations between daily activity and hemodynamic responses presented in a prior study (15), as well as in the current study, perturbations in chest wall volume regulation during treadmill walking at a speed corresponding to each individual patient's daily movement intensity. Accordingly, in the present study, patients were fitted with 89 retroreflective markers that were placed front and back over the chest wall, thereby allowing breath-by-breath measurement of total and compartmental (rib cage and abdominal) chest wall volume variations during 4-min constant-speed walking on the treadmill at a speed corresponding to a predetermined individualized daily movement intensity.

Study group. Eighteen consecutive patients with COPD across Global Initiative for Chronic Obstructive Lung Diseases (GOLD) stages II and III (Table 1) participated in the study according to certain inclusion criteria as follows: 1 ) a postbronchodilator forced expiratory volume in $1 \mathrm{~s}\left(\mathrm{FEV}_{1}\right)<70 \%$ predicted without significant reversibility $\left(<12 \%\right.$ change of the initial $\mathrm{FEV}_{1}$ value or $<200 \mathrm{ml}$ ) and 2) optimal medical therapy for at least $8 \mathrm{wk}$ with no change according to GOLD (24). Patients in the present study had previously participated in a study (15) investigating associations between daily physical activity levels and the magnitude of limitation in central and peripheral muscle hemodynamic capacities. That study (15) precluded concomitant investigation of the regulation in chest wall volume variations owing to the placement of 89 retroreflective markers on the chest wall (see below) that could interfere with the placement of chest electrodes to assess cardiac output by cardio-impedance. In the present study, 18 of the subjects had useable data, and they participated in this study, as well as the previous one (15), which had a hemodynamic rather than a ventilatory focus. Patients' characterization as active and less physically active was based on the median daily movement intensity $\left(1.88 \mathrm{~m} / \mathrm{s}^{2}\right)$ recorded during seven consecutive days for all 18 patients with COPD. The University Hospital Ethics Committee approved the study, and patients provided informed written consent (protocol number 18367).

Initial assessment. Patients underwent measurements for anthropometric indices, arterial blood gas, and pulmonary function parameters (i.e., spirometry, lung diffusion capacity, and static lung volumes). In brief, body mass index was calculated as the ratio of weight (in $\mathrm{kg}$ ) to height (in m) squared. Estimation of body composition was obtained by bioelectric impedance analysis using a specific device (Maltron BF 907; Maltron, Essex, UK). Fat-free mass index was obtained by dividing fat-free mass in kilograms by height in meters squared. Radial arterial tensions of $\mathrm{O}_{2}\left(\mathrm{~Pa}_{\mathrm{O}_{2}}\right)$ and $\mathrm{CO}_{2}\left(\mathrm{~Pa}_{\mathrm{CO}_{2}}\right)$ and arterial oxygen saturation $\left(\mathrm{Sa}_{\mathrm{O}_{2}}\right)$ were measured from 2-ml blood samples using a blood gas analyzer combined with a cooximeter (ABL 625; Radiometer, Copenhagen, Denmark). Spirometry and single-breath
Table 1. Patient clinical characteristics and anthropometric and pulmonary function data

\begin{tabular}{|c|c|c|c|}
\hline \multirow[b]{2}{*}{ Variables } & \multicolumn{3}{|c|}{ Movement Intensity of Daily Activity } \\
\hline & All & $\begin{array}{l}\text { Patients with } \\
<1.88 \mathrm{~m} / \mathrm{s}^{2}\end{array}$ & $\begin{array}{l}\text { Patients with } \\
\geq 1.88 \mathrm{~m} / \mathrm{s}^{2}\end{array}$ \\
\hline Number of patients & 18 & 9 & 9 \\
\hline Age, yr & $66 \pm 7$ & $71 \pm 3$ & $64 \pm 5^{*}$ \\
\hline Height, cm & $169 \pm 8$ & $170 \pm 6$ & $169 \pm 8$ \\
\hline Weight, $\mathrm{kg}$ & $74 \pm 11$ & $73 \pm 10$ & $75 \pm 12$ \\
\hline Body mass index, $\mathrm{kg} / \mathrm{m}^{2}$ & $25.7 \pm 4.4$ & $25.2 \pm 4.2$ & $26.1 \pm 4.0$ \\
\hline Fat free mass index, $\mathrm{kg} / \mathrm{m}^{2}$ & $18.1 \pm 1.3$ & $18.1 \pm 1.1$ & $18.2 \pm 1.3$ \\
\hline GOLD stage II/III & 9/9 & $4 / 5$ & $5 / 4$ \\
\hline $\mathrm{FEV}_{1}, 1$ & $1.42 \pm 0.58$ & $1.16 \pm 0.41$ & $1.59 \pm 0.64 *$ \\
\hline $\mathrm{FEV}_{1}, \%$ predicted & $48 \pm 17$ & $43 \pm 15$ & $53 \pm 16^{*}$ \\
\hline $\mathrm{FVC}, 1$ & $3.21 \pm 0.95$ & $2.80 \pm 0.63$ & $3.51 \pm 0.89^{*}$ \\
\hline FVC, $\%$ predicted & $88 \pm 21$ & $76 \pm 18$ & $97 \pm 19 *$ \\
\hline $\mathrm{FEV}_{1} / \mathrm{FVC}, \%$ & $42 \pm 9$ & $40 \pm 8$ & $42 \pm 10$ \\
\hline $\mathrm{IC}, 1$ & $2.29 \pm 0.67$ & $1.92 \pm 0.37$ & $2.55 \pm 0.70 *$ \\
\hline IC, $\%$ predicted & $74 \pm 20$ & $62 \pm 13$ & $83 \pm 17 *$ \\
\hline $\mathrm{RV}, 1$ & $4.25 \pm 0.93$ & $4.31 \pm 0.96$ & $4.21 \pm 0.91$ \\
\hline $\mathrm{RV}, \%$ predicted & $199 \pm 43$ & $184 \pm 35$ & $198 \pm 40$ \\
\hline FRC, 1 & $5.78 \pm 1.15$ & $5.78 \pm 1.04$ & $5.74 \pm 1.28$ \\
\hline FRC, \% predicted & $172 \pm 32$ & $167 \pm 23$ & $174 \pm 39$ \\
\hline TLC, 1 & $8.19 \pm 1.23$ & $7.91 \pm 1.24$ & $8.20 \pm 1.25$ \\
\hline TLC, $\%$ predicted & $129 \pm 19$ & $121 \pm 18$ & $134 \pm 18$ \\
\hline RV/TLC, \% predicted & $146 \pm 20$ & $151 \pm 18$ & $145 \pm 22$ \\
\hline $\mathrm{TL}_{\mathrm{CO}}, \%$ predicted & $55 \pm 19$ & $49 \pm 10$ & $61 \pm 11 *$ \\
\hline Resting $\mathrm{PaO}_{2}, \mathrm{mmHg}$ & $71 \pm 11$ & $65 \pm 7$ & $79 \pm 10^{*}$ \\
\hline Resting $\mathrm{PaCO}_{2}, \mathrm{mmHg}$ & $38 \pm 5$ & $41 \pm 4$ & $35 \pm 3^{*}$ \\
\hline Resting $\mathrm{SaO}_{2}, \%$ & $94 \pm 2$ & $93 \pm 2$ & $95 \pm 1 *$ \\
\hline
\end{tabular}

Values are expressed as means $\pm \mathrm{SD}$. $\mathrm{FEV}_{1}$, forced expiratory volume in $1 \mathrm{~s}$; FVC, forced vital capacity; IC, inspiratory capacity; RV, residual volume; FRC, functional residual capacity; TLC, total lung capacity; TL $_{\mathrm{CO}}$, diffusing capacity of the lung for carbon monoxide; $\mathrm{PaO}_{2}$, partial pressure of arterial oxygen; $\mathrm{PaCO}_{2}$, partial pressure of arterial carbon dioxide; $\mathrm{SaO}_{2}$, arterial oxygen saturation. *Significant differences between groups $(P<0.05)$.

transfer factor for carbon dioxide was measured according to the American Thoracic Society/European Respiratory Society standards (16) after administering $400 \mu \mathrm{g}$ salbutamol by a spirometer (Masterlab; Jaeger, Wurzburg, Germany). Postbronchodilator static lung volumes were assessed using whole-body plethysmography (Medgraphic Autolink 1085D; Medical Graphics, St. Paul, MN).

Assessment of daily movement intensity. Patients were equipped with an accelerometer DynaPort MoveMonitor (McRoberts BV, The Hague, The Netherlands) $(13,25,28)$ to assess for each individual the average daily movement intensity over seven consecutive days (2 weekend days and 5 weekdays). During an initial visit, patients while wearing the accelerometer performed an incremental exercise protocol on the treadmill (Track Motion, Kettler, Germany) to obtain a range of walking intensities at various walking speeds. Testing speed on the treadmill started at $1.4 \mathrm{~km} / \mathrm{h}$ and increased every 3 min by 0.8 $\mathrm{km} / \mathrm{h}$. For each speed, a mean walking intensity (expressed in $\mathrm{m} / \mathrm{s}^{2}$ ) was calculated from minute-by-minute data retrieved from the accelerometer. Subsequently, the mean walking intensity that corresponded to each individual patient's average daily movement intensity captured over 7 days was employed during an individualized 4-min constant-speed walking test on the treadmill, as described below.

Treadmill protocol at individualized speed. Within $72 \mathrm{~h}$ after the end of the monitoring period, patients visited the hospital for an individualized speed protocol on the treadmill. During this assessment, patients walked for $4 \mathrm{~min}$ at the predetermined speed captured during physical activity monitoring (i.e., at the individual patient's intensity). Before imposing the predetermined intensity, patients stood on the treadmill for $4 \mathrm{~min}$ for baseline measurements and then warmed up at a speed corresponding to $1.4 \mathrm{~km} / \mathrm{h}$ for an additional 4 $\min$. 
Cardiopulmonary measurements during the treadmill protocol. During the 4-min constant-speed treadmill protocol, pulmonary gas exchange and ventilatory variables were recorded breath by breath (Vmax 229; SensorMedics, Yorba Linda, CA). Inspiratory capacity (IC) maneuvers to establish the magnitude of dynamic lung hyperinflation were performed at frequent intervals. Patients were instructed after 4-6 regular tidal breaths to make maximal IC efforts from their end-expiratory lung volume to their total lung capacity (TLC) according to previously described methods $(18,19,35)$. Heart rate was determined using the R-R interval from a 12-lead electrocardiogram (Marquette Max; Marquette Hellige, Freiburg, Germany). Arterial oxyhemoglobin saturation was determined by pulse oximetry $\left(\mathrm{SpO}_{2} \%\right.$; Nonin 8600; Nonin Medical, Plymouth, $\left.\mathrm{MN}\right)$ with its analog signal being directed to the system.

Chest wall volume measurements during the treadmill protocol. During the 4-min constant-speed treadmill protocol, chest wall kinematics and compartmental volumes were recorded breath by breath by OEP (OEPsystem; BTS, Milano, Italy) (Fig. 1). The OEP device and its methodology have been thoroughly described elsewhere $(2,30)$. In brief, the movement of 89 retroreflective markers placed front and back over the chest wall from clavicles to pubis was recorded. Each marker was tracked by six calibrated video cameras (Smart System, BTS), three in front of the subject and three behind at a distance of 2.5 $\mathrm{m}$. Dedicated software reconstructs the three-dimensional coordinates of the markers in real time by stereo photogrammetry and calculates total and compartmental chest wall volume variations using Gauss's theorem. As in a previous study (12) that utilized the OEP technique during exercise, the duration of exercise at each patient's individualized walking intensity was $4 \mathrm{~min}$, to allow the patients to achieve a quasistable state so that the operational lung volumes could be accurately measured. All patients were carefully instructed not to grasp treadmill handles during standing and walking and to keep their arms away from the rib cage so that lateral markers could be visualized.

As in the study by Aliverti and coworkers (2), the chest wall was modeled as being composed of two compartments, the rib cage and the abdomen. Chest wall volume $\left(\mathrm{V}_{\mathrm{CW}}\right)$ was the sum of the rib cage volume $\left(\mathrm{V}_{\mathrm{RC}}\right)$ and the abdominal volume $\left(\mathrm{V}_{\mathrm{AB}}\right)$. Tidal volume measured by the OEP $\left(\mathrm{VT}_{\mathrm{CW}}\right)$ was calculated as the difference between

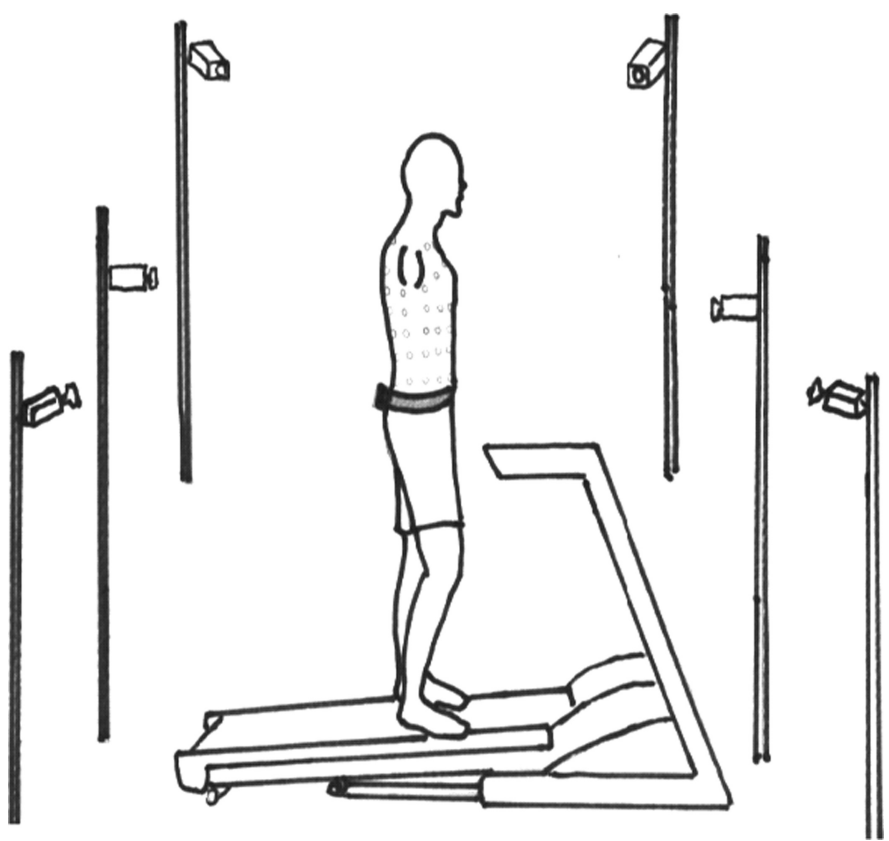

Fig. 1. Optoelectronic plethysmography. Chest wall reflective marker placement and the calibrated camera positioning during walking on the treadmill. end-inspiratory $\left(E I V_{C W}\right)$ and end-expiratory $\left(E E V_{C W}\right)$ chest wall volume. Chest wall volumes at TLC (TLCVcw) were determined during IC efforts. Inspiratory reserve chest wall volume $\left(I_{R V_{C W}}\right)$ was calculated as the difference between TLCV $\mathrm{CW}_{\mathrm{CW}}$ and EIV $\mathrm{CW}_{\mathrm{CW}}$. Total and compartmental end-expiratory and end-inspiratory chest wall volumes during the treadmill protocol were measured at the beginning and the end of inspiratory flow (zero-flow points) relative to that during quiet breathing. Symptom ratings were monitored every minute throughout walking test using the 1-10 Borg scale (5). During this protocol minute-by-minute movement intensity, expressed in $\mathrm{m} / \mathrm{s}^{2}$, was measured by the accelerometer to verify that the movement intensity reproduced for each individual patient during the 4-min treadmill protocol corresponded to his average movement intensity previously captured over 7 days. OEP data were analyzed at comparable levels of minute ventilation (i.e., isoventilation).

Statistical analysis. Data are reported as means \pm SD unless otherwise stated. Normal distribution was assessed using the ShapiroWilkes test. Differences between active and less active patients in baseline anthropometric and clinical characteristics and lung function variables as well as in chest wall volume variations at the highest level of minute ventilation were tested using unpaired $t$-test. Two-way ANOVA for repeated measures, followed by Bonferroni adjustment, was employed to identify significant differences in chest wall volume variations at comparable levels of minute ventilation (i.e., isoventilation) between active and less physically active patients. Pearson correlation coefficient analysis was performed to determine associations between patients' daily movement intensity and operational chest wall volume variations recorded at the highest isoventilation level in the group of the less physically active patients. To explore the level of agreement between outdoor average daily movement intensity captured during 7 days of monitoring and indoor movement intensity recorded during the treadmill protocol at each patient's individualized speed, a Bland-Altman plot was employed. Stepwise multiple regression analyses established the strongest significant contributors to daily movement intensity (dependent variable). Independent determinants of daily movement intensity included all significant demographic and baseline pulmonary function variables, as well as all significant operational chest wall volume parameters. In addition to investigating the likely interaction of changes in chest wall volumes and hemodynamic variables reported in our previous study (15), we employed a stepwise multiple-regression analysis as well. Data were analyzed using the SPSS statistical program, version 18 (SPSS, Chicago, IL). The level of significance was set at $P<0.05$.

\section{RESULTS}

Patient characteristics and functional capacity. Less physically active patients (mean daily movement intensity: $1.58 \pm$ $0.11 \mathrm{~m} / \mathrm{s}^{2}$ ) were older and had more severe airway obstruction, reduced diffusion capacity, and arterial oxygen tension compared with the more physically active patients (mean daily movement intensity: $2.00 \pm 0.13 \mathrm{~m} / \mathrm{s}^{2}$ ) (Table 1).

Reproduction of daily movement activity on the treadmill. The average movement intensity of each individual patient for the 7 days of monitoring (expressed in $\mathrm{m} / \mathrm{s}^{2}$ ) was highly correlated ( $r=0.96, P<0.001$ ) with the movement intensity reproduced for each individual patient during the 4-min constant-speed treadmill test (Fig. 2). The average movement intensity reproduced for each individual patient during the 4-min constant-speed treadmill protocol corresponded to a treadmill speed equivalent to $2.8 \pm 0.4 \mathrm{~km} / \mathrm{h}$ for the less active patients and $3.5 \pm 0.6 \mathrm{~km} / \mathrm{h}$ for the more active patients. Treadmill speeds were different between groups $(P<0.01)$. 

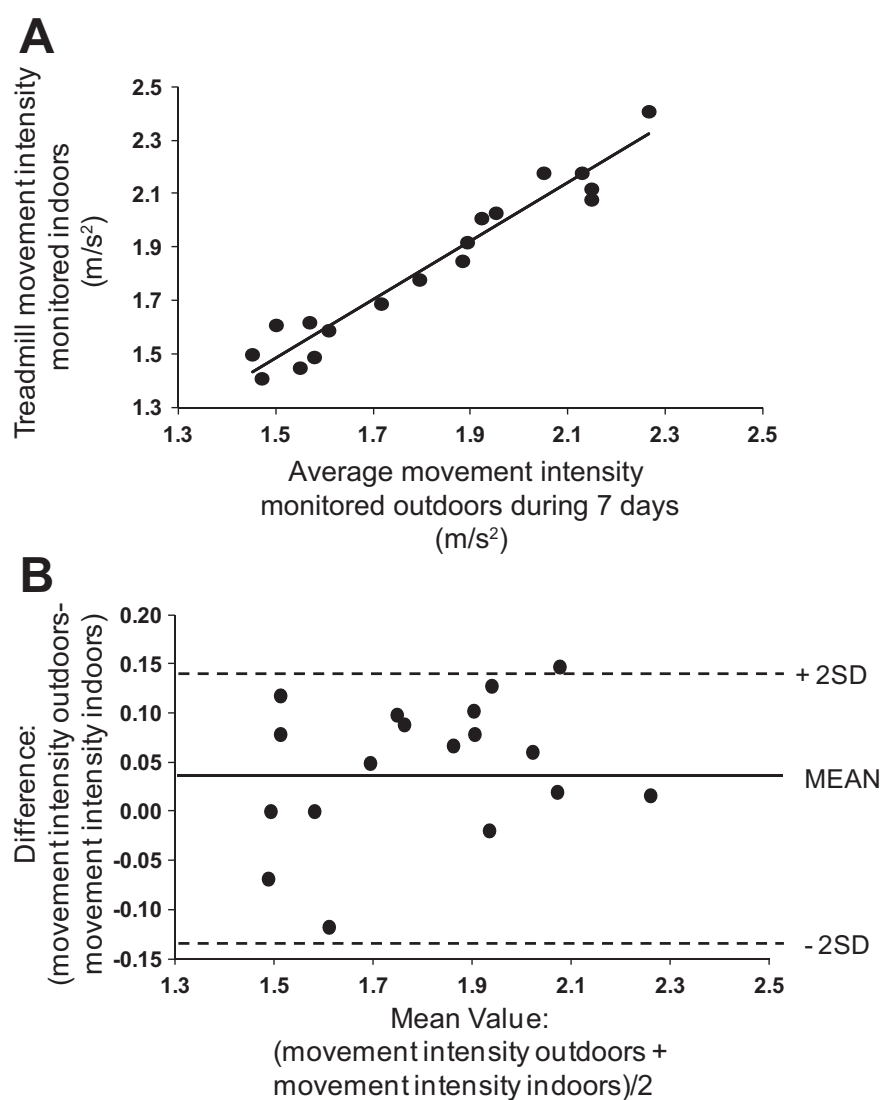

Fig. 2. Correlation coefficient (Pearson's $r$ ) between average daily movement intensity monitored outdoors during 7 days and the movement intensity reproduced indoors during the individualized speed exercise protocol on the treadmill $(A)$ and the Bland-Altman correspondent plot $(B)$.

Associations of baseline lung function and individualized movement intensity. Daily movement intensity was significantly associated with a number of baseline pulmonary function variables (Table 2). From all baseline pulmonary function variables (Table 2), the strongest significant contributor to daily movement intensity as selected by stepwise multiple regression analysis was diffusion capacity $\left(r^{2}=0.416, P=\right.$ $0.009)$.

Chest wall volume variations at individualized movement intensity. At comparable levels of minute ventilation throughout the 4-min constant-speed treadmill protocol, less physically active patients compared with the more active patients experienced significantly compromised tidal chest wall volume $\left(\mathrm{VT}_{\mathrm{CW}}\right)$ and inspiratory reserve chest wall volume $\left(\mathrm{IRV}_{\mathrm{CW}}\right)$ (Fig. 3, $A$ and $E$ ), a more rapid and shallow breathing pattern (Fig. 3D), lower arterial oxygen saturation $\left(\mathrm{SpO}_{2}\right)$ (Fig. 3), and greater intensity of breathlessness (Fig. $3 F$ ). In addition, at comparable levels of minute ventilation, end-expiratory chest wall volume $\left(\mathrm{EEV}_{\mathrm{CW}}\right)$ was lower in active compared with less active patients (Fig. $4 D$ ). This was due to lower end-expiratory rib cage and abdominal volumes in active compared with less active patients (Fig. 4, $E$ and $F$ ). No differences were found in total or compartmental end-inspiratory chest wall volumes at comparable levels of minute ventilation between active and less active patients (Fig. 4, $A-C$ ).

At peak isoventilation level (i.e., the highest minute ventilation recorded in the group of less physically active patients corresponding to a comparable level of minute ventilation in the group of more active patients), $\mathrm{VT}_{\mathrm{CW}}, \mathrm{IRV}_{\mathrm{CW}}, \mathrm{EEV} \mathrm{cw}$, breathing frequency, inspiratory and expiratory flow rates $\left(\mathrm{VT}_{\mathrm{CW}} / \mathrm{T}_{\mathrm{i}}\right.$ and $\mathrm{VT}_{\mathrm{CW}} / \mathrm{T}_{\mathrm{e}}$, respectively), dyspnea sensation, as well as $\mathrm{SpO}_{2}$ were significantly correlated with daily movement intensity captured for each individual patient (Table 3). In addition, at peak isoventilation level, the amount of dynamic lung hyperinflation, as assessed by changes from rest in IC, was significantly associated $(r=0.55 ; P=0.017)$ with the average movement intensity monitored for 7 days (Fig. 5). Furthermore, the magnitude of dynamic lung hyperinflation was significantly lower in active compared with the less physically active patients $(0.14 \pm 0.05$ vs. $0.29 \pm 0.061, P<0.05)$. Moreover, the stepwise multiple-regression analysis model including all significant respiratory variables of Table 3 (i.e., operational chest wall volume parameters, Borg dyspnea score, and $\mathrm{SpO}_{2}$ at the peak isoventilation level), as well as age retained $\mathrm{IRV}_{\mathrm{CW}}\left(r^{2}=0.420, P=0.014\right)$, expiratory flow $\left(r^{2}\right.$ change $=0.174, P=0.026)$, and Borg dyspnea score $\left(r^{2}\right.$ change $=0.123, P=0.041)$ as the significant best contributors accounting for $71.7 \%$ of the explained variance in daily movement intensity. The stepwise multiple-regression analysis model including all significant operational chest wall volume parameters at peak isoventilation level as well as cardiac output, quadriceps muscle oxygen saturation $\left(\mathrm{Sto}_{2}\right)$, arteriovenous oxygen content difference, and oxygen delivery from our previous published study (15) retained $\mathrm{Sto}_{2}\left(r^{2}=0.675\right.$, $P=0.001)$ and IRVcw $\left(r^{2}\right.$ change $\left.=0.102, P=0.029\right)$ as the significant best contributors, accounting for $77.7 \%$ of the explained variance in daily movement intensity.

\section{DISCUSSION}

The important finding of the present study is that more physically active patients with COPD exhibited a greater ability to expand tidal volume and to maintain adequate inspiratory reserve volume at exercise intensities and tasks commonly adopted in everyday life (Fig. 3). Additionally, when total and compartmental chest wall volumes were expressed relative to comparable levels of minute ventilation, active patients differed from the less active ones in terms of the lower magnitude of end-expiratory dynamic chest wall hyperinflation (Fig. 4) that was accomplished by significantly lower volume variations in both end-expiratory ribcage and abdominal compartments (Fig. 4).

Table 2. Correlation coefficient (Pearson's $r$ ) between each individual patient's average movement intensity of daily activity and demographic characteristics and lung function variables

\begin{tabular}{lrc}
\hline \multicolumn{1}{c}{ Variables } & \multicolumn{1}{c}{$r$} & $P$ Values \\
\hline Age, yr & -0.640 & 0.004 \\
FVC, 1 & 0.556 & 0.017 \\
FVC, \% predicted & 0.511 & 0.030 \\
FEV $/$ FVC, \% predicted & 0.536 & 0.025 \\
IC, 1 & 0.548 & 0.028 \\
IC, \% predicted & 0.650 & 0.001 \\
TLCO, \% predicted & 0.680 & 0.038 \\
Resting $\mathrm{PaO}_{2}, \mathrm{mmHg}$ & 0.429 & 0.050 \\
Resting $\mathrm{PaCO}_{2}, \mathrm{mmHg}$ & -0.524 & 0.045 \\
\hline
\end{tabular}

$\mathrm{FEV}_{1} / \mathrm{FVC}$ : Tiffeneau index. 

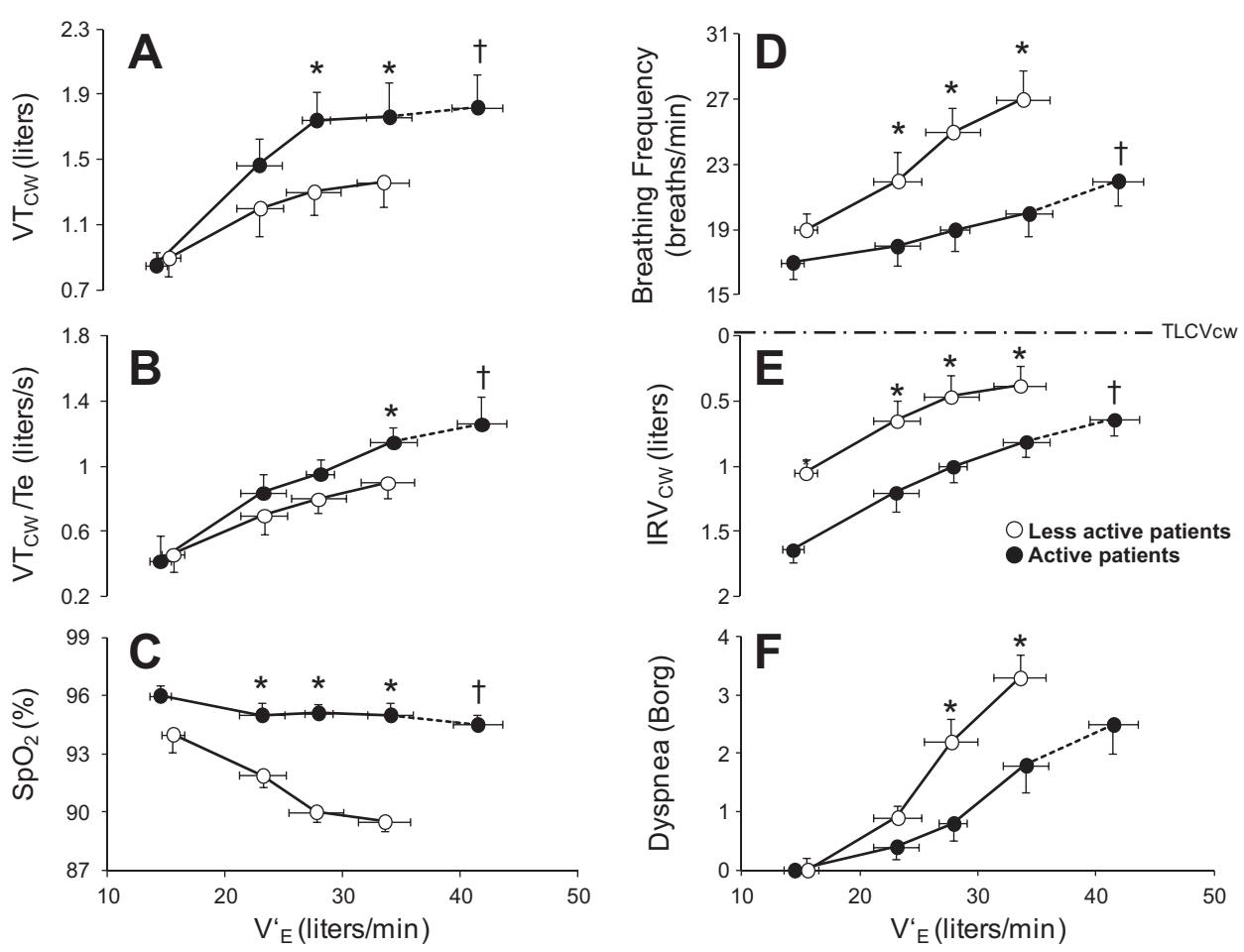

Fig. 3. Chest wall tidal volume $\left(\mathrm{VT}_{\mathrm{CW}}\right)(A)$, mean expiratory flow $\left(\mathrm{VT}_{\mathrm{CW}} / \mathrm{Te}\right)(B)$, arterial oxygen saturation $\left(\mathrm{SpO}_{2}\right)(c)$, breathing frequency $(D)$, inspiratory reserve chest wall volume $\left(\operatorname{IRV}_{\mathrm{CW}}\right)(E)$, and dyspnea as rated by the Borg scale $(F)$ in active $(\bullet)$ and less active patients $(\bigcirc)$ at comparable levels of minute ventilation $\left(\mathrm{V}_{\mathrm{E}}\right)$. Mean values and standard error bars are shown for the 2 groups. Peak values for operational chest wall volumes and other variables for the active patients corresponded to greater levels of minute ventilation during walking. $E$ : dashed line indicates chest wall volume at total lung capacity $\left(\mathrm{TLCV}_{\mathrm{CW}}\right)$. *Significant differences $(P<$ $0.05)$ between groups at comparable levels of minute ventilation (2-way ANOVA for repeated measures). $\uparrow$ Significant differences $(P<$ $0.05)$ between groups at the highest level of minute ventilation recorded for each group (unpaired $t$-test).
Novelties of the study. The present study has several important and novel approaches in the assessment of total and compartmental chest wall volumes (using OEP), employing movement intensities commonly observed in daily life (assessed by accelerometer) as well as reproducing those levels of movement intensities in the laboratory using common forms of exercise (e.g., walking).

Detailed analysis of variations in total and compartmental chest wall volumes at the same walking intensity engaged by patients in daily living is different from the well-controlled laboratory conditions previously reported $(2-4,27,29,31)$ engaging exclusively cycling exercise that hardly resembles the nature of daily physical activity. With such an approach, OEP allowed the calculation of inspiratory reserve chest wall volume that is known to be associated with the intensity of dyspnea sensations and exercise limitation $(19,21,22)$. In addition, to the best of our knowledge, the present study provides for the first time compartmental variations in chest wall volumes during walking in patients with COPD, thereby showing that rib cage and abdominal volume variations are compatible with those previously reported by others during cycling $(2,29,31)$. Furthermore, as OEP captures compartmental volume changes that may potentially reflect respiratory muscle contribution (1), we were able to express suggestions about abdominal muscle recruitment in physically active and less active patients with COPD.

Important determinants of daily physical activity levels. Accelerometers are increasingly used over the past few years to assess real life levels of physical activity in patients with $\operatorname{COPD}(11,23,25,28,34)$ and investigate associations between disease severity as well as physiological limitations with daily activity levels $(13,15)$. It is well recognized that the degree of lung function impairment limits intensity of daily physical activity $(6,9,15,33)$. Hence, it is conceivable that the more severely impaired patients will be less physically active in daily living $(23,34)$. We were able to confirm this by showing significant associations between intensity of daily physical activity with baseline pulmonary function variables (Table 2) and with chest wall volume variations measured at peak levels of minute ventilation recorded in the group of the less physically active patients during the treadmill test (Table 3). Furthermore, lung diffusion capacity, as an index of gasexchange inefficiency and disease severity, was a significant contributor of intolerance to intensity of physical activity. Additionally, most likely owing to greater baseline lung impairment (Table 1), less active patients were more limited in terms of inspiratory volume expansion, setting the scene for differences in breathing pattern (i.e.: tidal volume, breathing frequency, and expiratory flow rate) (Fig. 3).

Taking into consideration the findings of our previous study (15) suggesting that less physically active patients demonstrated compromised hemodynamic responses and increased arteriovenous oxygen difference, it is conceivable that this group might be limited by respiratory factors as well, thereby potentially impeding the capacity to secure adequate oxygen availability at the level of peripheral muscles. Thus, the present study expands earlier findings (15) by showing that the magnitude of exercise-induced dynamic lung and chest wall hyperinflation (Fig. 5) (Table 3) is also an important contributor to the level of daily physical activity in patients with COPD. The relative importance of central hemodynamic, respiratory, and peripheral muscle factors that compromise physical activity in active and less physically active patients has been addressed by investigating the interaction of changes among hemodynamic, ventilatory, and muscular factors. Our findings suggest that local muscle oxygen saturation and limitation in tidal volume expansion are the most significant contributors to the limitation of intensity of daily physical activity. 

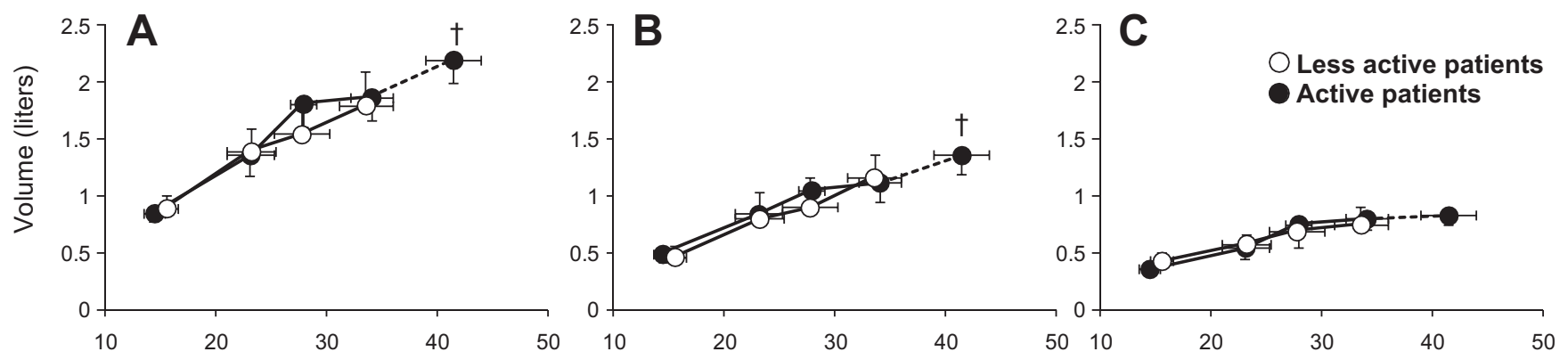

End-Expiratory chest wall

End-Expiratory rib cage

End-Expiratory abdomen
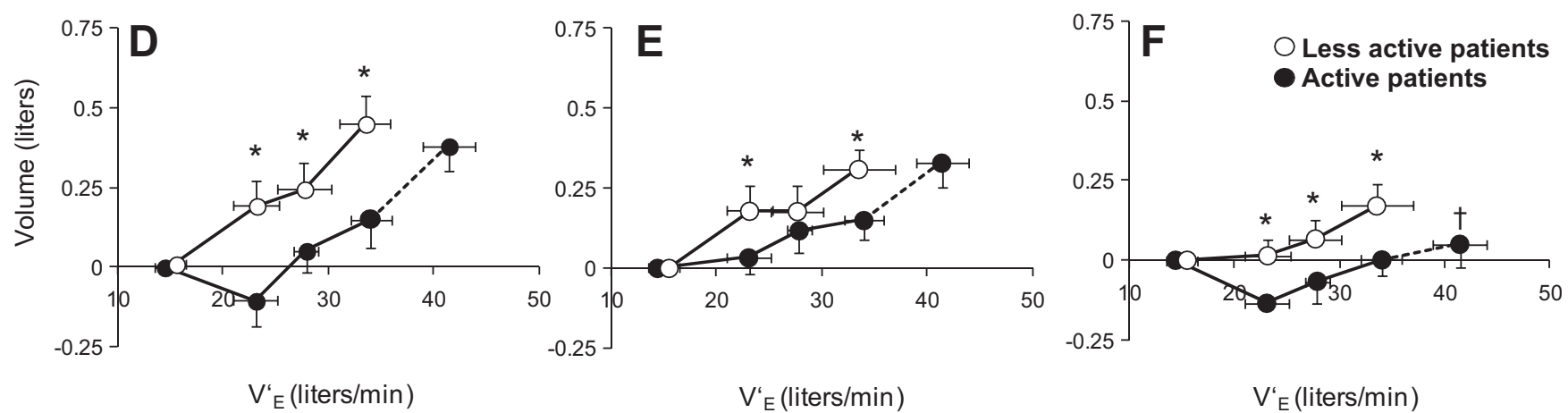

Fig. 4. Volume variations at end-inspiration $(A-C)$ and end-expiration $(D-F)$ of the total chest wall $(A$ and $D)$, the rib cage compartment $(B$ and $E)$, and the abdomen $(C$ and $F)$ plotted as a function of minute ventilation $\left(\mathrm{V}_{\mathrm{E}}\right)$ in active patients $(\bullet)$ and less active patients $(\mathrm{O})$. Mean values and standard error bars are shown for the 2 groups. Peak values for total and compartmental chest wall volumes and other variables for the active patients corresponded to greater levels of minute ventilation during walking. *Significant differences $(P<0.05)$ between groups at comparable levels of minute ventilation $(2$-way ANOVA for repeated measures). $†$ Significant differences $(P<0.05)$ between groups at the highest level of minute ventilation recorded for each group (unpaired $t$-test).

Chest wall volume variations and breathing pattern. Taking into account that 1 ) the rate of dynamic hyperinflation is primarily determined by both the extent of expiratory flow limitation and the degree of ventilatory demand (14) and that 2) there were differences between active and less active patients in terms of the individualized walking intensity on the treadmill, operational chest wall volumes were compared between

Table 3. Correlation coefficient (Pearson's $r$ ) between each individual patient's average movement intensity of daily physical activity and respiratory variables recorded at peak isoventilation level

\begin{tabular}{lrr}
\hline \multicolumn{1}{c}{ Variables } & \multicolumn{1}{c}{$r$} & $P$ Values \\
\hline $\mathrm{VT}_{\mathrm{CW}}, 1$ & 0.575 & 0.013 \\
$\mathrm{IRV}_{\mathrm{CW}}, 1$ & 0.651 & 0.003 \\
EEVcw, 1 & -0.648 & 0.004 \\
Breathing frequency, breaths/min & -0.488 & 0.040 \\
$\mathrm{VT}_{\mathrm{CW}} / \mathrm{Ti}, 1 / \mathrm{s}$ & 0.506 & 0.038 \\
$\mathrm{VT}_{\mathrm{CW}} / \mathrm{Te}, 1 / \mathrm{s}$ & 0.592 & 0.016 \\
$\mathrm{Borg}_{\text {dyspnea score }}$ & -0.504 & 0.033 \\
$\mathrm{SpO}_{2}, \%$ & 0.603 & 0.008 \\
\hline
\end{tabular}

Peak iso-ventilation level is the highest minute ventilation recorded in the group of less physically active patients corresponding to a comparable level of minute ventilation in the group of more active patients. $\mathrm{VT}_{\mathrm{CW}}$, chest wall tidal volume; IRV $\mathrm{CW}_{\mathrm{W}}$, chest wall inspiratory reserve volume; EEVcw, end-expiratory chest wall volume; $\mathrm{VT}_{\mathrm{CW}} / \mathrm{Ti}$, mean inspiratory flow; $\mathrm{VT}_{\mathrm{CW}} / \mathrm{Te}$, mean expiratory flow; $\mathrm{SpO}_{2}$, arterial oxygen saturation measured by pulse oximetry. active and less active patients at comparable levels of minute ventilation (i.e., at isoventilation). Accordingly, detailed analysis of chest wall volume variations and breathing pattern parameters at comparable minute ventilation levels during walking (Figs. 3 and 4) confirmed that the more active patients with COPD exhibited substantially higher expiratory flow rates (Fig. $3 B$ ) and lower degrees of dynamic chest wall hyperinflation (Fig. 4D), thereby allowing greater expansion of tidal volume (Fig. $3 A$ ) and adequate inspiratory reserve chest wall volume (Fig. $3 E$ ). Reduced chest wall dynamic hyperinflation and greater tidal volume expansion exhibited by the more active patients yielded significantly lower breathing frequency (Fig. 3D) and levels of dyspnea sensation (Fig. 3F), as well as milder changes in arterial oxygen saturation (Fig. $3 C$ ) compared with the less active patients.

Furthermore, it was shown that active patients demonstrated significantly lower end-expiratory abdominal hyperinflation at comparable levels of minute ventilation (Fig. $4 F$ ), attenuating further increases in total end-expiratory chest wall volume (Fig. 4D). In fact, this is a phenomenon presented in healthy elderly individuals (31) as well as in less impaired patients with COPD (e.g., those with GOLD stage II) during exercise (29, 31). The aforementioned findings are in accordance with the suggestion that, in patients with less severe COPD, abdominal muscle recruitment is likely to have an impact on the expiratory flow rate and on the degree of end-expiratory lung volume 


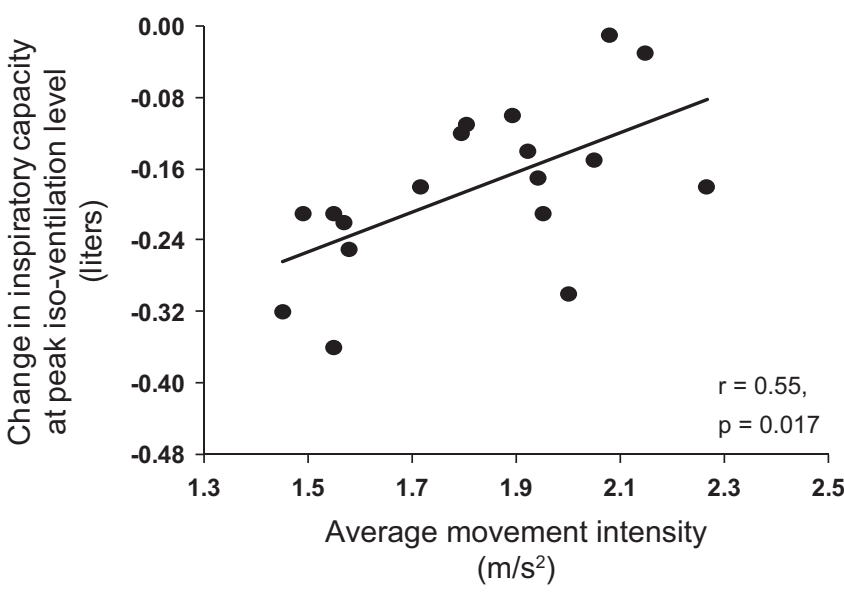

Fig. 5. Relationship (Pearson's $r$, correlation coefficient) between average movement intensity recorded by accelerometer outdoors during 7 days and change in inspiratory capacity (IC) in 18 patients with chronic obstructive pulmonary disease. Change in IC refers to change of this variable measured at peak isoventilation level (i.e., the highest minute ventilation recorded in the group of less physically active patients corresponding to a comparable level of minute ventilation in the group of more active patients) during the treadmill exercise compared with baseline (rest).

regulation (17). Our findings are further supported by previous studies reporting that expiratory abdominal muscle recruitment is associated with lower degrees of dynamic chest wall hyperinflation either after pharmacological (bronchodilators' administration) (3) or nonpharmacological (e.g., pulmonary rehabilitation) (10) interventions. In addition, the lower end-expiratory chest wall volume variations in the more active patients were mainly attributed to changes of the abdominal and not the rib cage compartment $(3,10)$ (Fig. 4, D-F). Interestingly, in line with previous studies $(3,10)$, it is apparent that the pattern of dynamic rib cage hyperinflation is highly comparable in both active and less physically active patients, whereas the abdominal compartment undergoes a different pattern in terms of end-expiratory chest wall volume variation (Fig. 4).

OEP assesses changes in end-expiratory chest wall volume (including lung volumes, gas compression, and decompression in the lungs and blood shifts between the lung and extremities), which differs from the well-implemented method to assess end-expiratory dynamic lung hyperinflation by repeated IC maneuvers at regular intervals during exercise. Similarly to previous studies $(2,27,29,31)$ showing that, during cycling, end-expiratory chest wall volume is regulated differently in patients with COPD with varying degrees of lung dysfunction, we have demonstrated that, in less active patients, end-expiratory volume increased progressively (from $\sim 0.2$ to $\sim 0.51$ ), whereas, in active patients, end-expiratory volume decreased at low levels of ventilatory requirement and then progressively increased (from $\sim-0.1$ to $\sim 0.4$ 1) (Fig. 3D). Indeed, previous findings $(14,29)$ have indicated that variations in the magnitude of exercise-induced end-expiratory dynamic hyperinflation are associated with profound variations in the degree of expiratory flow limitation and expiratory flow, and this might be the case in our patients with COPD.

The new feature of this study is that the more physically active patients were able to increase tidal volume by decreasing end-expiratory abdominal volume, maintaining a safe "distance" between the chest wall volume at TLC and that at the end of tidal inspiration, namely the inspiratory reserve chest wall volume. An inadequate inspiratory reserve volume occurs when this volume reaches minimal levels (about 0.35 1) (19, 21 ), thereby intensifying dyspnea sensations. Indeed, at comparable levels of minute ventilation, active patients had a large inspiratory reserve volume amounting to $\sim 0.651$ (7.6\% of their TLCcw) (Fig. 3E) compared with the less active patients, who did not maintain their inspiratory reserve chest wall volume above the so-called minimal levels (19-22). The lower inspiratory reserve chest wall volume in less active patients was translated into a tachypneic and shallow breathing pattern (Fig. 3D).

Collectively, the findings of the present and previous studies $(9,15)$ suggest that physiological limitations imposed by impaired respiratory and gas-exchange mechanisms are not entirely responsible for the reduced physical activity levels documented in COPD (34). Indeed, if such physiological inadequacies were the sole factor in explaining daily activity limitation, both active and less active patients would have experienced comparable magnitudes of ventilatory restriction (i.e., IRVcw and breathing frequency) and $\mathrm{SpO}_{2}$ at the respective intensities of daily movement. Hence, several behavioral and habitual factors may also play a contributing role in determining daily physical activity in COPD (34).

Taking in consideration that compromised respiratory function, in terms of the magnitude of dynamic lung hyperinflation (9) and of limitation in both tidal and inspiratory reserve volume presented in this study, affects the level of physical activity in patients with COPD, therapeutic interventions (e.g., pharmacological), whose aim is to lower the magnitude of dynamic hyperinflation and to increase inspiratory reserve volume, are justified in promoting higher daily activity levels.

In conclusion, the inability to maintain adequate inspiratory reserves, the magnitude of expiratory flow limitation, and the accompanying degree of dynamic chest wall hyperinflation emerge as the most important ventilatory contributors to the intensity of physical activity adopted by COPD in daily living. Consequently, patients who exhibit greater ability to expand tidal volume and to maintain adequate inspiratory reserve volume tend to be more physically active. It is thus anticipated that interventions aimed at mitigating limitations in chest wall volumes would enhance daily physical activity levels in COPD through an increase in the intensity of movement.

\section{ACKNOWLEDGMENTS}

An abstract of this work entitled "Mechanical restriction to tidal volume expansion partially determines intensity of physical activity in COPD" received the Excellence Grant in Clinical Physiology and Exercise by the Clinical Physiology, Sleep and Pulmonary Circulation Assembly during the 2013 Annual European Respiratory Society Congress in Barcelona.

\section{GRANTS}

This work was supported by the Innovative Medicines Initiative Joint Undertaking (IMU-JU no. 115011) through the Thorax Foundation.

\section{DISCLOSURES}

No conflicts of interest, financial or otherwise, are declared by the authors.

\section{AUTHOR CONTRIBUTIONS}

Author contributions: E.A.K., A. Aliverti, Z.L., S.G.Z., and I.V. conception and design of research; E.A.K., A. Aliverti, Z.L., M.V., I.N., and A. Asimakos performed experiments; E.A.K., A. Aliverti, and M.V. analyzed data; E.A.K., 
A. Aliverti, M.V., I.N., S.G.Z., and I.V. interpreted results of experiments; E.A.K. and Z.L. prepared figures; E.A.K., A. Aliverti, Z.L., I.N., A. Asimakos, S.G.Z., and I.V. drafted manuscript; E.A.K., A. Aliverti, Z.L., M.V., I.N., A. Asimakos, S.G.Z., and I.V. edited and revised manuscript; E.A.K., A. Aliverti, Z.L., M.V., I.N., A. Asimakos, S.G.Z., and I.V. approved final version of manuscript.

\section{REFERENCES}

1. Aliverti A, Cala SJ, Duranti R, Ferrigno G, Kenyon CM, Pedotti A, Scano G, Sliwinski P, Macklem PT, Yan S. Human respiratory muscle actions and control during exercise. J Appl Physiol 83: 1256-1269, 1997.

2. Aliverti A, Stevenson N, Dellaca RL, Lo Mauro A, Pedotti A, Calverley PMA. Regional chest wall volumes during exercise in chronic obstructive pulmonary disease. Thorax 59: 210-216, 2004.

3. Aliverti A, Rodger K, Dellaca RL, Lo Mauro A, Pedotti A, Calverley PMA. Effect of salbutamol on lung function and chest wall volumes at rest and during exercise in COPD. Thorax 60: 916-924, 2005.

4. Aliverti A, Quaranta M, Chakrabarti B, Albuquerque ALP, Calverley PM. Paradoxical movement of the lower ribcage at rest and during exercise in COPD patients. Eur Respir J 33: 49-60, 2009.

5. Borg GA. Physiological bases of perceived exertion. Med Sci Sports Exerc 14: 377-381, 1982.

6. Bossenbroek L, de Greef MH, Wempe JB, Krijnen WP, Ten Hacken NH. Daily physical activity in patients with chronic obstructive pulmonary disease: a systematic review. COPD 8: 306-319, 2011.

7. Diaz O, Villafranca C, Chezzo H, Borzone G, Leiva A, Milic-Emil J, Lisboa C. Role of inspiratory capacity on exercise tolerance in COPD patients with and without tidal expiratory flow limitation at rest. Eur Respir J 16: 269-275, 2000.

8. Diaz O, Villafranca C, Ghezzo H, Borzone G, Leiva A, Millic-Emili J, Lisboa C. Breathing pattern and gas exchange at peak exercise in COPD patients with and without tidal flow limitation at rest. Eur Respir $J$ 17: 1120-1127, 2001.

9. Garcia-Rio F, Lores V, Mediano O, Rojo B, Hernanz A, LopezCollazo E, Alvarez-Sala R. Daily physical activity in patients with chronic obstructive pulmonary disease is mainly associated with dynamic hyperinflation. Am J Respir Crit Care Med 180: 506-512, 2009.

10. Georgiadou O, Vogiatzis I, Stratakos G, Koutsoukou A, Golemati S, Aliverti A, Roussos C, Zakynthinos S. Effects of rehabilitation on chest wall volume regulation during exercise in COPD patients. Eur Respir J 29: 284-291, 2007.

11. Hernandes NA, Teixeira Dde C, Probst VS, Brunetto AF, Ramos EM, Pitta F. Profile of the level of physical activity in the daily lives of patients with COPD in Brazil. J Bras Pneumol 35: 949-956, 2009.

12. Iandelli I, Aliverti A, Kayser B, Dellacà R, Cala S, Duranti R, Kelly S, Scano G, Sliwinski P, Yan S, Macklem PT, Pedotti A. Determinant of exercise performance in normal men with externally imposed expiratory flow limitation. J Appl Physiol 92: 1943-1952, 2002.

13. Kortianou EA, Louvaris Z, Vasilopoulou M, Nasis I, Kaltsakas G, Koulouris NG, Vogiatzis I. Activity monitoring reflects cardiovascular and metabolic variations in COPD patients across GOLD stages II to IV. Respir Physiol Neurobiol 189: 513-520, 2013.

14. Koulouris NG, Dimopoulou I, Valta P, Finkelstein R, Cosio MG, Milic-Emili J. Detection of expiratory flow limitation during exercise in COPD patients. J Appl Physiol 82: 723-731, 1997.

15. Louvaris Z, Kortianou EA, Spetsioti S, Vasilopoulou M, Nasis I, Asimakos A, Zakynthinos S, Vogiatzis I. Intensity of daily physical activity is associated with central hemodynamic and leg muscle oxygen availability in COPD. J Appl Physiol 115: 794-802, 2013.

16. Miller MR, Hankinson J, Brusasco V, Burgos F, Casaburi R, Coates A, Crapo R, Enright P, van der Grinten CP, Gustafsson P, Jensen R, Johnson DC, MacIntyre N, McKay R, Navajas D, Peterson OF, Pellegrino R, Viegi G, Wagner J. Standardisation of spirometry. Eur Respir J 26: 319-338, 2005.

17. Ninane V, Rypens F, Yernault JC, De Troyer A. Abdominal muscle use during breathing in patients with chronic airflow obstruction. Am Rev Respir Dis 146: 16-21, 1992.
18. O'Donnell DE, Webb KA. Exertional breathlessness in patients with chronic airflow limitation: The role of lung hyperinflation. Am J Respir Crit Care Med 148: 1351-1357, 1993.

19. O'Donnell DE, Revill SM, Webb KA. Dynamic hyperinflation and exercise intolerance in chronic obstructive pulmonary disease. Am J Respir Crit Care Med 164: 770-777, 2001.

20. O'Donnell DE, Fluge T, Gerken F, Hamilton A, Webb K, Aguilaniu B, Make B, Magnussen H. Effects of tiotropium on lung hyperinflation, dyspnea and exercise tolerance in COPD. Eur Respir J 23: 832-840, 2004.

21. O'Donnell DE, Hamilton AL, Webb KA. Sensory-mechanical relationships during high-intensity, constant-work-rate exercise in COPD. J Appl Physiol 101: 1025-1035, 2006.

22. Peters MM, Webb KA, O'Donnell DE. Combined physiological effects of bronchodilators and hyperoxia on exertional dyspnea in normoxic COPD. Thorax 61: 559-567, 2006

23. Pitta F, Troosters T, Spruit MA, Probst VS, Decramer M, Gosselink R. Characteristics of physical activities in daily life in chronic obstructive pulmonary disease. Am J Respir Crit Care Med 171: 972-977, 2005.

24. Rabe KF, Hurd S, Anzueto A, Barnes PJ, Buist SA, Calverley P, Fukuchi Y, Jenkins C, Rodriguez-Roisin R, van Weel C, Zielinski J. Global strategy for the diagnosis, management, and prevention of chronic obstructive pulmonary disease: GOLD executive summary. Am J Respir Crit Care Med 176: 532-555, 2007.

25. Rabinovich RA, Louvaris Z, Raste Y, Langer D, Remoortel HV, Giavedoni S, Burtin C, Regueiro EM, Vogiatzis I, Hopkinson NS, Polkey MI, Wilson FJ, Macnee W, Westerterp KR, Troosters T. Validity of physical activity monitors during daily life in patients with COPD. Eur Respir J 42: 1205-1215, 2013.

26. Shrikrishna D, Patel M, Tanner RJ, Seymour JM, Connolly BA, Puthucheary ZA, Walsh SL, Bloch SA, Sidhu PS, Hart N, Kemp PR, Moxham J, Polkey MI, Hopkinson NS. Quadriceps wasting and physical inactivity in patients with COPD. Eur Respir J 40: 1115-1122, 2012.

27. Takara LS, Cunha TM, Barbosa P, Rodrigues MK, Oliveira MF, Nery LE, Neder JA. Dynamics of chest wall volume regulation during constant work rate exercise in patients with chronic obstructive pulmonary disease. Braz J Med Biol Res 45: 1276-1283, 2012.

28. Van Remoortel H, Raste Y, Louvaris Z, Giavedoni S, Burtin C, Langer D, Wilson F, Rabinovich R, Vogiatzis I, Hopkinson NS, Troosters T. Validity of six activity monitors in chronic obstructive pulmonary disease: A comparison with indirect calorimetry. PLoS One 7: e39198, 2012.

29. Vogiatzis I, Georgiadou O, Golemati S, Aliverti A, Kosmas E, Kastanakis E, Geladas N, Koutsoukou A, Nanas S, Zakynthinos S, Roussos Ch. Patterns of dynamic hyperinflation during exercise and recovery in patients with severe chronic obstructive pulmonary disease. Eur Respir $J$ 60: 723-729, 2005.

30. Vogiatzis I, Aliverti A, Golemati S, Georgiadou O, Lo Mauro A, Kosmas E, Kastanakis E, Roussos Ch. Respiratory kinematics by optoelectronic plethysmography during exercise in men and women. Eur J Appl Physiol 93: 581-587, 2005.

31. Vogiatzis I, Stratakos G, Athanasopoulos D, Georgiadou O, Golemati S, Koutsoukou A, Weisman I, Roussos Ch Zakynthinos S. Chest wall volume regulation during exercise in COPD patients with GOLD stages II to IV. Eur Respir J 32: 42-52, 2008.

32. Vogiatzis I, Zakynthinos S. Factors limiting exercise tolerance in chronic lung diseases. Compr Physiol 2: 1779-1817, 2012.

33. Walker PP, Burnett A, Flavahan PW, Calverley PM. Lower limb activity and its determinants in COPD. Thorax 63: 683-689, 2008.

34. Watz H, Pitta F, Rochester CL, Garcia-Aymerich J, ZuWallack R, Troosters T, Vaes AW, Puhan MA, Jehn M, Polkey MI, Vogiatzis I, Clini EM, Toth M, Gimeno-Santos E, Waschki B, Esteban C, Hayot M, Casaburi R, Porszasz J, McAuley E, Singh SJ, Langer D, Wouters EF, Magnussen H, Spruit MA. An official European Respiratory Society statement on physical activity in COPD. Eur Respir J. 2014 Oct 30. pii: erj00468-2014. [Epub ahead of print].

35. Yan S, Kaminski D, Sliwinsky P. Reliability of inspiratory capacity for estimating end-expiratory lung volume changes during exercise in patients with chronic obstructive pulmonary disease. Am J Respir Crit Care Med 156: 55-59, 1997. 